\title{
Insights into species diversity of associated crustose coralline algae (Corallinophycidae, Rhodophyta) with Atlantic European maerl beds using DNA barcoding
}

\author{
Cristina Pardo*, Ignacio Bárbara ${ }^{1}$, Rodolfo Barreiro ${ }^{2} \&$ Viviana Peña ${ }^{3}$ \\ Grupo BioCost, Dpto. de Bioloxía, Facultade de Ciencias, Univ. da Coruña, 15071 A Coruña, Spain \\ "Corresponding author: cristina.pardo.carabias@udc.es, http://orcid.org/0000-0002-2534-3961 \\ IIgnacio.barbara@udc.es, http://orcid.org/0000-0003-1779-0224 \\ 2rodolfo.barreiro@udc.es, http://orcid.org/0000-0002-6049-5215 \\ ${ }^{3}$ vpena@udc.es, http://orcid.org/0000-0001-7003-3850
}

\begin{abstract}
DNA barcoding in combination with morpho-anatomical analysis was applied to study the diversity of crustose coralline algae associated to two maer beds from two protected Atlantic European areas from Brittany and Galicia -France and Spain, respectively-. Given the records of gametophytes of the maerl species Phymatolithon calcareum under crustose growth-forms, and that associated crustose coralline algae appear to be involved in the recruitment of new maerl plants, we compared the species composition between the associated crustose coralline algae to Breton and Galician maerl beds with the maerl species identified in these beds in previous DNA barcoding surveys. Our molecular results revealed higher species diversity in associated crustose coralline algae than in maerl-forming species. Nine taxa of crustose coralline algae were found in both study areas: four in Brittany and five in Galicia. Three species from Brittany were identified as Phymatolithon calcareum, Phymatolithon lamii, and Lithophyllum hibernicum. The remaining six ones were assigned to the genera Phymatolithon and Mesophyllum, along with Lithothamnion and Lithophyllum. Morpho-anatomical examination of diagnostic characters corroborated our molecular identification. Our results showed that the most representative genus of crustose coralline algae in Brittany was Phymatolithon, while in Galicia was Mesophyllum. In Brittany, Phymatolithon calcareum was found under both growth-forms, maerl and crustose coralline algae, the latter assigned to the gametophyte stage by the presence of uniporate conceptacles. The recruitment of new maerl plants involving associated crustose coralline algae with maerl beds may occur, but only we can affirm it for Phymatolithon calcareum in Brittany. By contrast, the different species composition between both growth-forms in the Galician maerl beds would indicate that the fragmentation of own free-living maerl species appears to be the most common propagation mechanism.
\end{abstract}

Keywords. Brittany, COI-5P, Corallinales, crustose coralline algae, diversity, Galicia, Hapalidiales, Lithophyllum, Lithothamnion, maerl, Mesophyllum, morphology, Phymatolithon, reproduction, rhodolith, SEM.
Resumen. En este trabajo se han utilizado el sistema de códigos de barras genéticos y análisis morfo-anatómicos para estudiar la diversidad de algas coralinas costrosas asociadas a dos fondos de maerl localizados en dos áreas protegidas del Atlántico Europeo en Bretaña y Galicia - Francia and España, respectivamente-. Dadas las citas recientes de gametófitos de la especie típica de lechos de maerl, Phymatolithon calcareum, bajo una forma de crecimiento costrosa, y que las algas coralinas incrustantes parecen estar implicadas en el reclutamiento de nuevas plantas del maerl, en este trabajo se compara la composición entre estas algas asociadas a fondos de maerl bretones y gallegos, con las especies del maerl identificadas en estos fondos en estudios previos de códigos de barras genéticos. Los resultados moleculares del presente trabajo revelaron una diversidad más alta en las algas coralinas costrosas asociadas que en las propias especies formadoras del maerl. En las áreas estudiadas se encontraron nueve táxones de algas coralinas costrosas: cuatro en la Bretaña francesa y cinco en Galicia. Tres especies de la Bretaña fueron identificadas como Phymatolithon calcareum, Phymatolithon lamii y Lithophyllum hibernicum. Las seis especies restantes fueron asignadas a los géneros Phymatolithon y Mesophyllum, junto con Lithothamnion y Lithophyllum. El examen morfo-anatómico de caracteres diagnósticos concordó con la identificación molecular. Los resultados obtenidos evidenciaron que el género más representativo de algas coralinas costrosas en la Bretaña fue Phymatolithon, mientras que en Galicia fue Mesophyllum. En la Bretaña, Phymatolithon calcareum fue encontrado bajo ambas formas de crecimiento - maerl y morfología incrustante-, en este último caso asignada a la generación gametofítica por la presencia de conceptáculos uniporados. El reclutamiento de nuevas plantas del maerl a través de las algas coralinas costrosas asociadas a fondos de maerl puede suceder, pero solo se puede afirmar para Phymatolithon calcareum en la Bretaña. Por contra, en los fondos de maerl de Galicia la composición diferente de ambas formas de crecimiento podría indicar que la fragmentación de las propias especies del maerl podría ser el mecanismo de propagación más común.

Palabras clave. Algas coralinas incrustantes, Bretaña, COI-5P, Corallinales, diversidad, Galicia, Hapalidiales, Lithophyllum, Lithothamnion, maerl, Mesophyllum, morfología, Phymatolithon, reproducción, rodolito, SEM.

Pardo C., Bárbara I., Barreiro R. \& Peña V. 2017. Insights into species diversity of associated crustose coralline algae (Corallinophycidae, Rhodophyta) with Atlantic European maerl beds using DNA barcoding. Anales Jard. Bot. Madrid 74 (2): e059. http://dx.doi.org/10.3989/ajbm.2459

Título en español: Conocimiento de la diversidad específica de algas coralinas costrosas (Corallinophycidae, Rhodophyta) asociadas a lechos de maerl de la Europa atlántica mediante la utilización de códigos de barras genéticos.

Received: 4-XI-2016; accepted: 23-V-2017; published online: 20-X-2017; Associate Editor: Antonio Flores.

\section{INTRODUCTION}

Atlantic European rhodolith beds, also known as maerl beds, are marine coastal habitats of high diversity composed of unattached non-geniculate coralline red algae - maerl/rhodolith - mixed with gravel, shells, and pebbles overgrown by crustose coralline algae (CCA; v.gr., Cabioch 1969, 1970; Bosence 1976; Peña \& Bárbara 2009; Hall-Spencer \& al. 2010; Adey \& al. 2015). The life-history of coralline red algae involves an alternation among three phases: a haploid gametophyte, a diploid carposporophyte — carried by the haploid female gametophyte after fertilization of carpogonium - , and a diploid tetrasporophyte (Irvine \& Chamberlain 1994). In two major Atlantic European maerl species, Phymatolithon calcareum (Pallas) W.H.Adey \& D.L.McKibbin and Lithothamnion corallioides (P.L.Crouan \& H.M.Crouan) P.L.Crouan \& H.M.Crouan, sporangial conceptacles have been occasionally reported - usually in free-living 
growth forms, and rarely in encrusting plants-, while gametangial conceptacles were even more rare (i.e., sexual conceptacles), and were only found as associated CCA in maerl beds from Brittany (Cabioch 1969, 1970; Adey \& McKibbin 1970; Maggs 1983; Woelkerling \& Irvine 1986; Irvine \& Chamberlain 1994; Mendoza \& Cabioch 1998; Peña \& Bárbara 2004; Peña \& al. 2014). Several authors (Lemoine 1910; Cabioch 1969, 1970; Freiwald 1995; Mendoza \& Cabioch 1998) have pointed out the contribution of the CCA to the development of further unattached maerl plants: after germination of the carpospores produced in CCA, juvenile tetrasporophytic plants grow also as crusts, and afterwards erect branches formed by these crusts break off, and give rise to unattached maerl plants. In Breton maerl beds, this type of recruitment was reported as the dominant (Cabioch 1969, 1970; Mendoza \& Cabioch 1998). Nonetheless, others authors (Bosence 1976; Woelkerling 1988; Peña \& al. 2014) have reported that fragmentation of free-living maerl thalli is the main method of propagation in the maerl beds.

In recent years, several molecular studies have been focused on the diversity and systematics of maerl-forming species in Atlantic Europe (Carro \& al. 2014; HernándezKantún \& al. 2014, 2015a, 2015b; Pardo \& al. 2014; Peña \& al. 2015b). However, the diversity and specific composition of their associated CCA are little-known. In Brittany, two DNA barcoding works found crustose plants of Phymatolithon calcareum and Phymatolithon lamii (Me.Lemoine) Y.M.Chamb., which showed that species composition of associated CCA could share some similarities with unattached maerl plants (Peña \& al. 2014, 2015b). Based on previous results obtained for maerlforming species of two study areas from Brittany and Galicia (Pardo \& al. 2014), we identified their associated CCA using a combination of DNA barcoding - COI-5Pand morpho-anatomical features. Apart from the species diversity of associated CCA, we compared the composition of both growth-forms (maerl and CCA) in order to know if the associated CCA could be involved in the recruitment of new unattached maerl plants.

\section{MATERIAL AND METHODS}

\section{Sampling collection}

A total of 16 CCA specimens, all of them epilithic over pebbles, were collected in two Atlantic European maerl beds in 2011 (table 1, fig. 1): Molène Archipelago -Brittany, France - and Ons Archipelago - Galicia, Spain - . Both archipelagos are located in two protected marine areas: Parc Naturel Marin d'Iroise - PNMI- and Parque Nacional Marítimo Terrestre das Illas Atlánticas de Galicia -PNMTIAG-, respectively. Collections were carried out by dredging at $<10 \mathrm{~m}$ in Brittany, and SCUBA diving at $13 \mathrm{~m}$ in Galicia, as complementary to maerl- forming species surveys (Carro \& al. 2014; Pardo \& al. 2014; Peña \& al. 2014). After collection, material was airdried, and vouchered in silica. Specimens were observed and photographed under stereomicroscope, and were deposited in the herbarium SANT (Thiers 2016; table 1).

\section{Molecular studies}

For the DNA extraction, a subsample was obtained by grounding of a small portion of each plant selected, choosing free epiphytes areas under stereomicroscope. DNA was extracted and purified using the DNeasy ${ }^{\circledR}$ Blood \& Tissue Kit - Qiagen, Valencia, CA - following manufacture's recommendations. A fragment of $664 \mathrm{bp}$ of the 5' end of the mitochondrial gene cytochrome oxidase I - COI$5 \mathrm{P}$ - was amplified using primers GazF1 and GazR1 (Saunders 2005). Amplifications PCR were performed in a Biometra TProfesional Basic thermocycler following Saunders \& McDevit (2012). Amplification success was evaluated by running the reactions in agarose gels. After excess of primers and nucleotides were removed with shrimp alkaline phosphatase and exonuclease I enzymes. PCR products were sequenced using the Sanger method at Macrogen facilities - http://www.macrogen.comThe sequences obtained were checked, edited and aligned with the program Geneious v. 5.6.6 - Biomatters, New Zealand-, and lodged in BOLD and GenBank (table 1). Haplotypes obtained were searched for matches in BOLD and GenBank databases to their molecular taxonomic identification (table 2). For the molecular analyses, publicly available COI-5P sequences for both maerl-forming and CCA taxa, particularly from Atlantic Europe, were included (Carro \& al. 2014; Pardo \& al. 2014; Peña \& al. 2014, 2015a, 2015b; Hernández-Kantún \& al. 2015a), as well as a sequence of Corallina officinalis L. as outgroup (Pardo \& al. 2015; table 3). In total, 30 sequences were used to generate a Maximum Likelihood - ML - tree. A bootstrap with 1,000 replicates was applied in Mega v. 6.0 with defaults settings (Tamura $\&$ al. 2013), using the General Time-Reversible model, Gamma distributed with Invariant sites - $\mathrm{GTR}+\mathrm{G}+\mathrm{I}$-, identified previously in ModelTest v. 2.1.3 (Darriba \& al. 2012) as the best-fitting substitution model using an Akaike Information Criterion - AIC — and Bayesian Information Criterion - BIC.

\section{Morphological studies}

CCA selected for molecular studies were examined under stereomicroscope, and representative fragments were examined by Scanning Electron Microscope - SEM, model JEOL JSM 6400, Univ. da Coruña-. Vegetative and reproductive features considered diagnostic and anatomical terminology followed Irvine \& Chamberlain (1994). 
Table 1. Information and collection data of the epilithic CCA collected in Brittany and Galicia.

\begin{tabular}{|c|c|c|c|c|}
\hline Haplotype ML-tree & Species identification & Voucher & $\begin{array}{l}\text { BOLD Process ID } \\
\text { GenBank accession no. } \\
\text { SANT-Algae }\end{array}$ & Collection details \\
\hline CCA_hap-1 & Phymatolithon calcareum & VPF00132/CPVP-945 & $\begin{array}{l}\text { CCOR002-17 } \\
\text { MF133371 } \\
\text { SANT-Algae } 29484\end{array}$ & $\begin{array}{l}\text { Molène Archipelago } \\
\text { Depth }<10 \mathrm{~m} \\
13-\mathrm{V}-2011 \\
48^{\circ} 23.150^{\prime} \mathrm{N}, 04^{\circ} 51.233^{\prime} \mathrm{W}\end{array}$ \\
\hline CCA_hap-2 & Phymatolithon sp. 5 & CPVP-758 & $\begin{array}{l}\text { CCOR001-17 } \\
\text { MF133370 } \\
\text { SANT-Algae } 00273\end{array}$ & $\begin{array}{l}\text { Ons Archipelago } \\
\text { Depth } 13 \mathrm{~m} \\
07-\mathrm{IV}-2011 \\
42^{\circ} 23.678^{\prime} \mathrm{N}, 08^{\circ} 54.915^{\prime} \mathrm{W}\end{array}$ \\
\hline \multirow{4}{*}{ CCA_hap-3 } & \multirow{4}{*}{ Phymatolithon sp. 6} & VPF00407B & $\begin{array}{l}\text { CCOR003-17 } \\
\text { MF133369 } \\
\text { SANT-Algae } 29168\end{array}$ & \multirow{4}{*}{$\begin{array}{l}\text { Molène Archipelago } \\
\text { Depth < } 10 \mathrm{~m} \\
13-\mathrm{V}-2011 \\
48^{\circ} 23.150^{\prime} \mathrm{N}, 04^{\circ} 51.233^{\prime} \mathrm{W}\end{array}$} \\
\hline & & VPF00407C & $\begin{array}{l}\text { CCOR004-17 } \\
\text { MF133366 } \\
\text { SANT-Algae } 29168\end{array}$ & \\
\hline & & VPF00411B & $\begin{array}{l}\text { CCOR005-17 } \\
\text { MF133368 } \\
\text { SANT-Algae } 29169\end{array}$ & \\
\hline & & VPF00406A & $\begin{array}{l}\text { CCOR006-17 } \\
\text { MF133367 } \\
\text { SANT-Algae } 00272\end{array}$ & \\
\hline CCA_hap-4 & Phymatolithon lamii & VPF00410 & $\begin{array}{l}\text { CCOR007-17 } \\
\text { MF133372 } \\
\text { SANT-Algae } 29485\end{array}$ & $\begin{array}{l}\text { Molène Archipelago } \\
\text { Depth }<10 \mathrm{~m} \\
13-\mathrm{V}-2011 \\
48^{\circ} 23.150^{\prime} \mathrm{N}, 04^{\circ} 51.233^{\prime} \mathrm{W}\end{array}$ \\
\hline CCA_hap-5 & Lithothamnion sp. 2 & VPF00483 & $\begin{array}{l}\text { CCOR008-17 } \\
\text { MF133360 } \\
\text { SANT-Algae } 00274 \\
\text { CCOR009-17 } \\
\text { MF133361 } \\
\text { SANT-Algae } 00275\end{array}$ & $\begin{array}{l}\text { Ons Archipelago } \\
\text { Depth } 13 \mathrm{~m} \\
\text { 7-IV-2011 } \\
42^{\circ} 23.678^{\prime} \mathrm{N}, 08^{\circ} 54.915^{\prime} \mathrm{W}\end{array}$ \\
\hline CCA_hap-7 & Mesophyllum sp. 3 & VPF00479 & $\begin{array}{l}\text { CCOR010-17 } \\
\text { MF133363 } \\
\text { SANT-Algae } 00276 \\
\text { CCOR011-17 } \\
\text { MF133362 } \\
\text { SANT-Algae } 00277\end{array}$ & $\begin{array}{l}\text { Ons Archipelago } \\
\text { Depth } 13 \mathrm{~m} \\
\text { 7-IV-2011 } \\
42^{\circ} 23.678^{\prime} \mathrm{N}, 08^{\circ} 54.915^{\prime} \mathrm{W}\end{array}$ \\
\hline CCA_hap-10 & Mesophyllum sp. 4 & VPF00480 & $\begin{array}{l}\text { CCOR012-17 } \\
\text { MF133365 } \\
\text { SANT-Algae } 00278 \\
\text { CCOR013-17 } \\
\text { MF133364 } \\
\text { SANT-Algae } 00279\end{array}$ & $\begin{array}{l}\text { Ons Archipelago } \\
\text { Depth } 13 \mathrm{~m} \\
7-\mathrm{IV}-2011 \\
42^{\circ} 23.678^{\prime} \mathrm{N}, 08^{\circ} 54.915^{\prime} \mathrm{W}\end{array}$ \\
\hline CCA_hap-11 & Lithophyllum sp. 3 & CPVP-762 & $\begin{array}{l}\text { CCOR014-17 } \\
\text { MF133359 } \\
\text { SANT-Algae } 00280\end{array}$ & $\begin{array}{l}\text { Ons Archipelago } \\
\text { Depth } 13 \mathrm{~m} \\
7-\mathrm{IV}-2011 \\
42^{\circ} 23.678^{\prime} \mathrm{N}, 08^{\circ} 54.915^{\prime} \mathrm{W}\end{array}$ \\
\hline CCA_hap-12 & Lithophyllum hibernicum & VPF00411A & $\begin{array}{l}\text { CCOR015-17 } \\
\text { KR733526 } \\
\text { SANT-Algae } 29169 \\
\text { CCOR016-17 } \\
\text { KR733456 } \\
\text { SANT-Algae 29168 }\end{array}$ & $\begin{array}{l}\text { Molène Archipelago } \\
\text { Depth }<10 \mathrm{~m} \\
\text { Date: } 13-\mathrm{V}-2011 \\
48^{\circ} 23.150^{\prime} \mathrm{N}, 04^{\circ} 51.233^{\prime} \mathrm{W}\end{array}$ \\
\hline
\end{tabular}

\section{RESULTS}

\section{Molecular results}

COI-5P sequences were obtained for the $16 \mathrm{CCA}$ specimens collected (table 1). They were grouped in 13 haplotypes -CCA_hap-1 to CCA_hap-13-: five from Molène Archipelago and eight from Ons Archipelago (table 1). These 13 haplotypes were delimited in nine CCA species belonging to four genera (fig. 2): Phymatolithon sp. 5 -CCA_hap-2-, Phymatolithon sp. 6 - CCA hap-3-, Phymatolithon calcareum -CCA_hap-1-, 


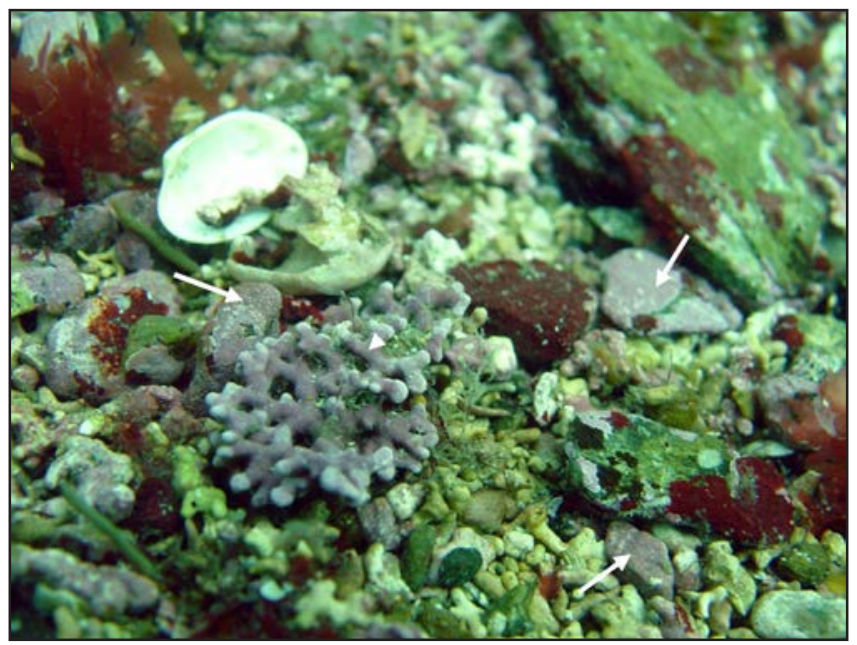

Fig. 1. Close up of the maerl bed studied in Ons Archipelago. [Arrow: epilithic CCA; arrowhead: a maerl-forming species.]

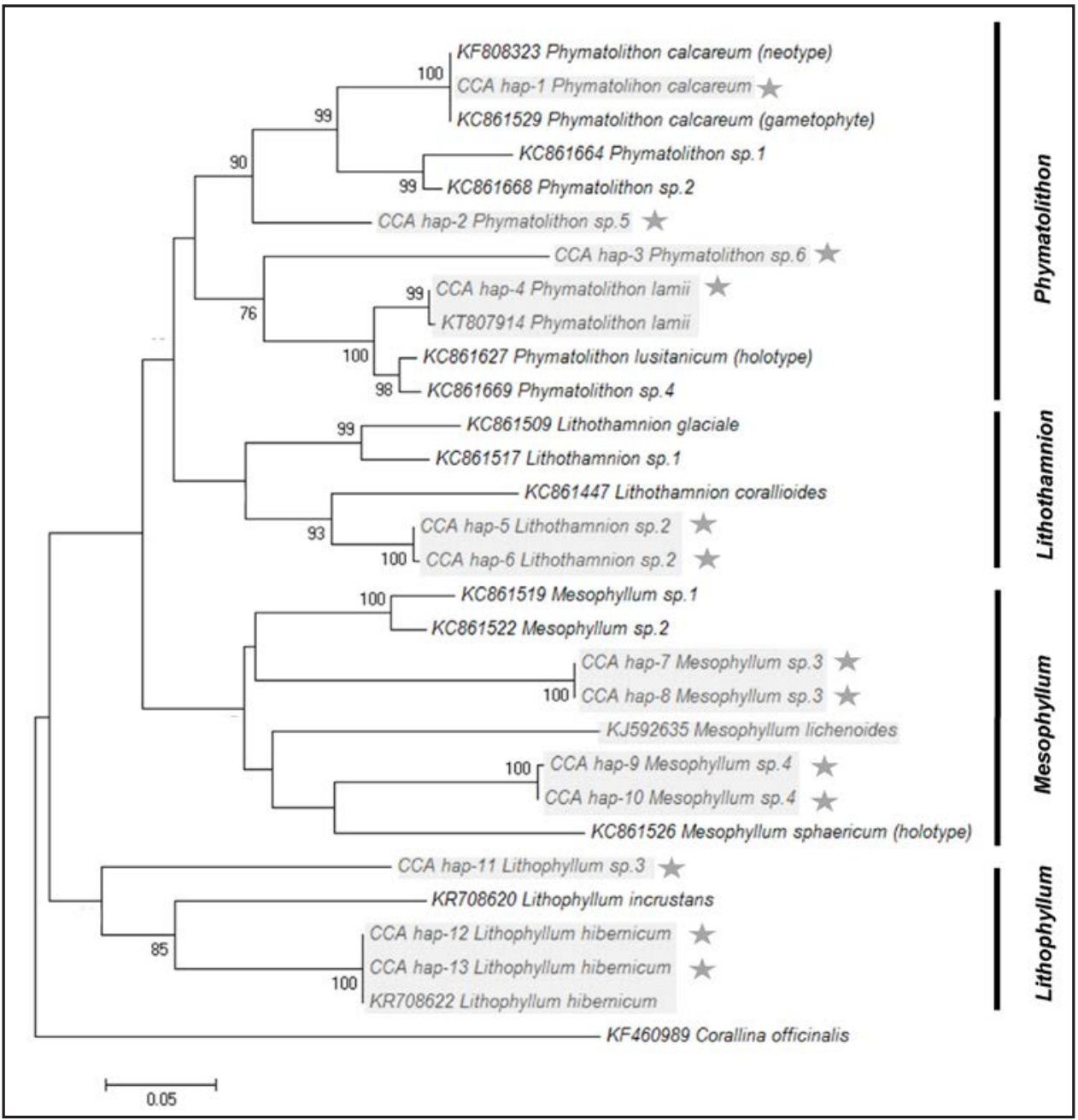

Fig. 2. Maximum-Likelihood - ML — tree for the haplotypes - i.e., DNA barcodes, in grey colour with a star- of the CCA associated to maerl beds from Ons Archipelago and Molène Archipelago showing relationships with other sequences of genus Lithophyllum, Phymatolithon, Lithothamnion, and Mesophyllum. [Encrusting growth-forms are indicated in grey colour; bootstrap support values $>75 \%$ - 1,000 replicates - are shown; outgroup: Corallina officinalis L.; scale bar refers to base subtitutions per site.] 


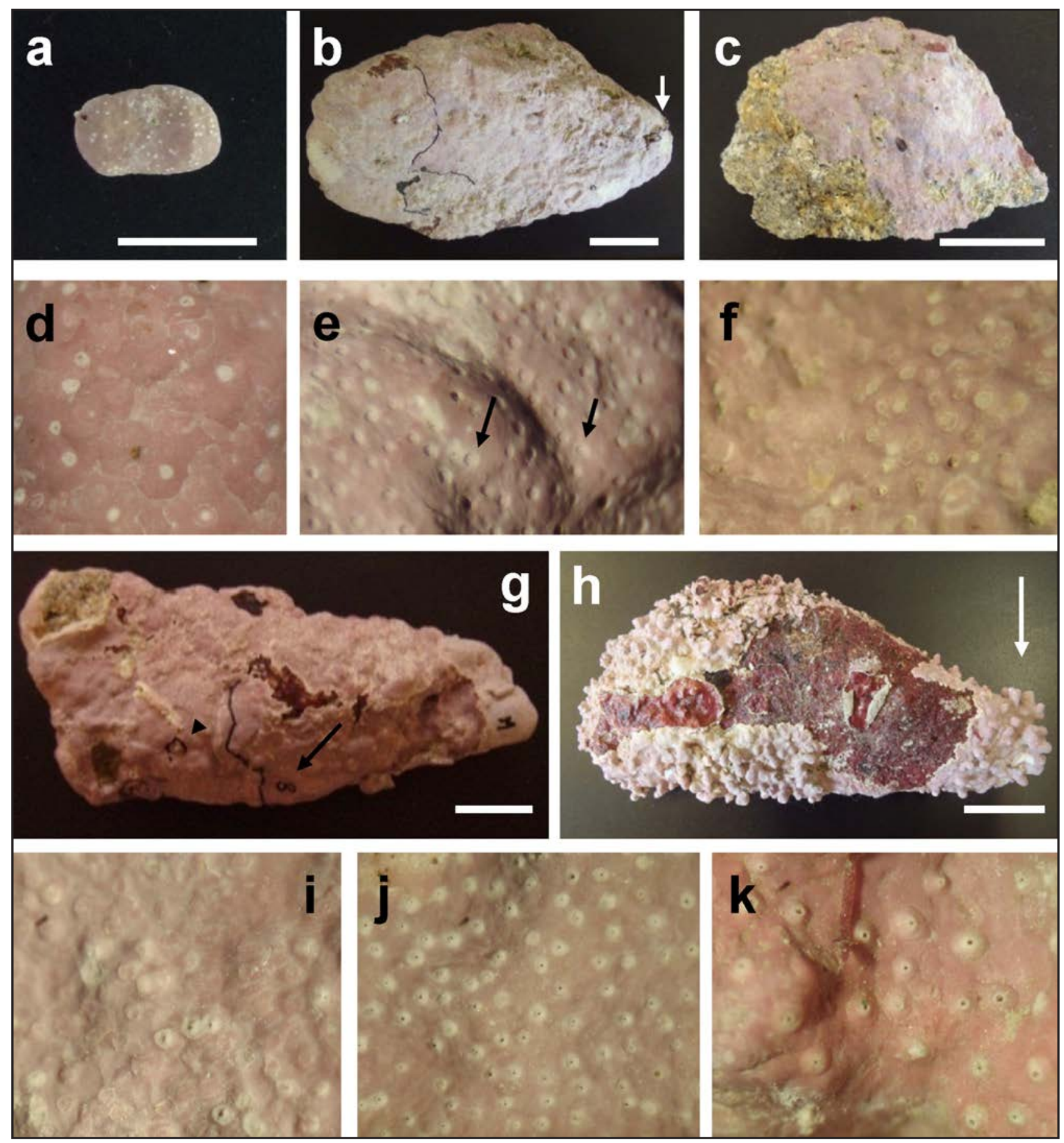

Fig. 3. Epilithic crusts associated with maerl beds in Galicia and in Brittany: a, Phymatolithon sp. 5 from Ons Archipelago (CPVP-758); b, Phymatolithon sp. 6 from Molène Archipelago (VPF00406A); c, Phymatolithon lamii from Molène Archipelago (VPF00410); d, Phymatolithon sp. 5 with asexual multiporate conceptacles (CPVP-758); e, Phymatolithon sp. 6 with sexual uniporate conceptacles (VPF00406A); f, Phymatolithon lamii with asexual multiporate conceptacles (VPF00410); g, Phymatolithon sp. 6 from Molène Archipelago (VPF00407B arrow, and VPF00407C arrowhead); h, Phymatolithon calcareum from Molène Archipelago (VPF00132); i, Phymatolithon sp. 6 with sexual uniporate conceptacles (VPF00407B); j, Phymatolithon sp. 6 with sexual uniporate conceptacles (VPF00407C); k, Phymatolithon calcareum with sexual uniporate conceptacles (VPF00132). [Scale bars: a, 1cm; b, c, h, $2.5 \mathrm{~cm} ; \mathrm{g}, 1.5 \mathrm{~cm}$. 
Table 2. Matches with our sequences in public databases (BOLD and GenBank). Only hits with an identity beyond a minimum threshold are reported in this table (98\%).

\begin{tabular}{|c|c|c|c|c|c|c|c|c|}
\hline \multirow{2}{*}{$\begin{array}{l}\text { Haplotype } \\
\text { ML-tree }\end{array}$} & \multicolumn{3}{|c|}{ BOLD } & \multicolumn{3}{|c|}{ GenBank } & \multirow[b]{2}{*}{ Taxonomy } & \multirow[b]{2}{*}{ Locality } \\
\hline & Number & Identity & Taxonomy & Locality & Accession no. & Identity & & \\
\hline CCA_hap-1 & BOLD:ACD0016 & $100 \%$ & $\begin{array}{l}\text { Phymatolithon } \\
\text { calcareum }\end{array}$ & $\begin{array}{l}\text { Falmouth } \\
\text { (Brithish } \\
\text { Isles) }\end{array}$ & KC861551 & $99 \%$ & $\begin{array}{l}\text { Phymatolithon } \\
\text { calcareum }\end{array}$ & $\begin{array}{c}\text { Galicia } \\
\text { (NW } \\
\text { Spain) }\end{array}$ \\
\hline CCA_hap-3 & $\begin{array}{l}\text { BOLD Process ID: } \\
\text { ABMMC9521-10 } \\
\text { BOLD Sample ID: } \\
\text { GWS006296 }\end{array}$ & $100 \%$ & $\begin{array}{l}\text { Phymatolithon } \\
\text { sp.3AT crust }\end{array}$ & $\begin{array}{c}\text { New } \\
\text { Brunswick } \\
\text { (Atlantic } \\
\text { Canada) }\end{array}$ & No match & - & - & - \\
\hline CCA_hap-4 & $\begin{array}{l}\text { BOLD Process ID: } \\
\text { NGCOR259-15 } \\
\text { BOLD Process ID: } \\
\text { VPF00075 }\end{array}$ & $100 \%$ & $\begin{array}{c}\text { Phymatolithon } \\
\text { lamii }\end{array}$ & $\begin{array}{l}\text { Brittany } \\
\text { (NW } \\
\text { France) }\end{array}$ & No match & - & - & - \\
\hline CCA_hap-12 & $\begin{array}{l}\text { BOLD Process ID: } \\
\text { NGCOR158-14 }\end{array}$ & $99.85 \%$ & Lithophyllum & Brittany & KR733526 & $100 \%$ & Lithophyllum & Brittany \\
\hline CCA_hap-13 & $\begin{array}{l}\text { BOLD Process ID: } \\
\text { VPF } 00230\end{array}$ & $100 \%$ & hibernicum & $\begin{array}{c}(\mathrm{NW} \\
\text { France) }\end{array}$ & KR733438 & $100 \%$ & hibernicum & $\begin{array}{c}(\mathrm{NW} \\
\text { France) }\end{array}$ \\
\hline
\end{tabular}

Table 3. COI-5P sequences downloaded from GenBank and BOLD used in the ML tree (fig. 2).

\begin{tabular}{|c|c|c|}
\hline Species & GenBank accession no. & BOLD Process ID \\
\hline Lithophyllum incrustans & KR708620 & - \\
\hline Lithophyllum hibernicum & KR708622 & - \\
\hline $\begin{array}{l}\text { Phymatolithon calcareum } \\
\text { neotype, maerl plant }\end{array}$ & KF808323 & MAERL237-13 \\
\hline $\begin{array}{l}\text { Phymatolithon calcareum } \\
\text { gametophyte, encrusting plant }\end{array}$ & KC861529 & MAERL235-13 \\
\hline $\begin{array}{l}\text { Phymatolithon sp. } 1 \\
\text { maerl plant }\end{array}$ & KC861664 & MAERL069-11 \\
\hline $\begin{array}{l}\text { Phymatolithon sp. } 2 \\
\text { maerl plant }\end{array}$ & KC861668 & MAERL067-11 \\
\hline $\begin{array}{l}\text { Phymatolithon lamii } \\
\text { encrusting plant }\end{array}$ & KT807914 & - \\
\hline $\begin{array}{l}\text { Phymatolithon lusitanicum } \\
\text { holotype, maerl plant }\end{array}$ & KC861627 & MAERL086-11 \\
\hline $\begin{array}{l}\text { Phymatolithon sp. } 4 \\
\text { maerl plant }\end{array}$ & KC861669 & MAERL087-11 \\
\hline $\begin{array}{l}\text { Lithothamnion glaciale } \\
\text { maerl plant }\end{array}$ & KC861509 & MAERL224-13 \\
\hline $\begin{array}{l}\text { Lithothamnion sp. } 1 \\
\text { maerl plant }\end{array}$ & KC861517 & MAERL011-11 \\
\hline $\begin{array}{l}\text { Lithothamnion corallioides } \\
\text { maerl plant }\end{array}$ & KC861447 & MAERL017-11 \\
\hline $\begin{array}{l}\text { Mesophyllum sp. } 1 \\
\text { maerl plant }\end{array}$ & KC861519 & MAERL019-11 \\
\hline $\begin{array}{l}\text { Mesophyllum sp. } 2 \\
\text { maerl plant }\end{array}$ & KC861522 & MAERL217-13 \\
\hline $\begin{array}{l}\text { Mesophyllum lichenoides } \\
\text { encrusting plant }\end{array}$ & KJ592635 & - \\
\hline $\begin{array}{l}\text { Mesophyllum sphaericum } \\
\text { holotype, maerl plant }\end{array}$ & KC861526 & MAERL015-11 \\
\hline Corallina officinalis & KF460989 & COR006-12 \\
\hline
\end{tabular}


Phymatolithon lamii _CCA_hap-4-, Lithothamnion sp. 2 -CCA_hap-5 and 6, both differing in $1 \mathrm{bp}-$, Mesophyllum sp. 3 -CCA_hap-7 and 8, both differing in $1 \mathrm{bp}-$, Mesophyllum sp. $\overline{4}$-CCA_hap-9 and 10, both differing in $1 \mathrm{bp}$-, Lithophyllum hibernicum - CCA hap-12 and 13-, and Lithophyllum sp. 3 -CCA_hap11- (figs. 3, 4).

Four CCA species were detected in the Breton maerl bed, and were successfully identified by matching with BOLD and GenBank databases (table 2): Phymatolithon calcareum, Phymatolithon lamii, and Lithophyllum hibernicum. Likewise, the species named as Phymatolithon sp. 6 -CCA_hap-3; table 1) scored with Phymatolithon sp. 3AT crust from NW Atlantic - Canada - . By contrast, the Galician CCA haplotypes did not match with any available sequence, but according to the ML-tree performed they were resolved into five different taxa (fig. 2): Phymatolithon sp. 5, Lithothamnion sp. 2, Mesophyllum sp. 3, Mesophyllum sp. 4, and Lithophyllum sp. 3.

\section{Morpho-anatomical examination}

Phymatolithon sp. 5 (fig. 3a) showed domed epithallial cells, and subepithallial cells as short as cells subtending them (fig. 5a); cell fusions between contiguous filaments were common (fig. 5b); multiporate asexual conceptacles showed white pore plates (fig. 3d), surrounded by rim and with roof slightly depressed (fig. 5c). Phymatolithon sp. 6 (figs. 3b, e, g, i, j, 4q) and Phymatolithon calcareum (fig. 3h, k) showed sexual uniporate conceptacles, white coloured in surface view, flushed with thallus surface to sunken in Phymatolithon sp. 6, while raised in Phymatolithon calcareum. Phymatolithon lamii (fig. 3c, f) showed asexual multiporate conceptacles with white pore plates flushed with thallus surface or slightly sunken.

Lithothamnion sp. 2 (fig. 4a, b) showed epithallial cells mainly flared and somewhat flattened, subepithallial cells as long or longer than cells subtending them (fig. 5d, e). Cell fusions between cells of contiguous filaments were common (fig. 5f); asexual multiporate conceptacles with sunken pore plate and white in colour (fig. $4 \mathrm{~d}$, e).

Mesophyllum sp. 3 (fig. 4c, g) showed epithallial cells somewhat flared, with subepithallial cells longer than cells subtending them (fig. 6d); cell fusions between contiguous filaments (fig. 6e); multiporate asexual conceptacles with sunken pore plates (figs. 4f, k, 6a); conceptacle chambers were elliptical, $90 \mu \mathrm{m}$ height by $137 \mu \mathrm{m}$ in diameter, and conceptacle roof of $11 \mu \mathrm{m}$ thickness (fig. 6b, c). Mesophyllum sp. 4 (fig. 4h, j) showed epithallial cells somewhat flared, subepithallial cells as long as or longer than cells subtending them, and cell fusions common between contiguous filaments (fig. 6f); uniporate sexual conceptacles were prominent on thallus surface (fig. 41, n); multiporate asexual conceptacles showed sunken pore plates (fig. 41, n), and also raised poro plates at the same time in the plant CPVP-769 (fig. 4n); conceptacle chambers were elliptical, $77 \mu \mathrm{m}$ height by $189 \mu \mathrm{m}$ in diameter, and conceptacle roof of $40 \mu \mathrm{m}$ thickness (fig. $6 \mathrm{~g}, \mathrm{~h}$ ).

Lithophyllum hibernicum (fig. 4o-q), and Lithophyllum sp. 3 (fig. 4i, m), showed uniporate conceptacles slightly flushed with thallus surface.

\section{DISCUSSION}

In our study, nine CCA species were recorded associated with two Atlantic European maerl beds: Molène -Brittany - and Ons - Galicia - Archipelagos. Despite that these CCA collections consisted on epilithic crustose corallines growing partial or entirely over pebbles, we are aware that could be interpreted as "rhodolith" given that this term is extensively applied to unattached nodules with non-algal core (Irvine \& Chamberlain 1994; v.gr. see fig. 6 in Basso \& al. 2009). While the four CCA species associated with the Breton maerl bed matched with available sequences from northern Atlantic coast - Phymatolithon calcareum, Phymatolithon lamii, Lithophyllum hibernicum, and Phymatolithon sp. 3ATcrust-, the five CCA taxa from the Galician maerl bed did not obtain any record. However, according to the molecular and morphological data obtained, CCA collected in Galicia were identified up to genus level as Phymatolithon sp. 5, Lithothamnion sp. 2, Mesophyllum sp. 3, Mesophyllum sp. 4, and Lithophyllum sp. 3. The absence of any match with public databases containing more than 4,000 COI-5P sequences of coralline red algae pointed out the cryptic diversity still uncovered in coralline algae.

The nine taxa delimited corresponded to the same four maerl-forming genera in Atlantic Europe -Lithothamnion, Phymatolithon, Mesophyllum, and Lithophyllum (Irvine \& Chamberlain 1994; Pardo \& al. 2014; Hernández-Kantún \& al. 2015a)_. Nevertheless, the number of associated CCA species recorded was higher than the number of maerl species cited in both studied areas (Carro \& al. 2014; Pardo \& al. 2014; Peña \& al. 2014, 2015b): four CCA vs. two maerl species in Molène Archipelago - Phymatolithon calcareum and Lithothamnion corallioides-; and five CCA vs. three maerl species in Ons Archipelago Phymatolithon calcareum, Phymatolithon lusitanicum, and Lithothamnion corallioides-. The similarity in the species composition between the both growth-forms is approximately $25 \%$ in the Breton maerl bed, but totally dissimilar - $0 \%$ shared species — in the Galician bed.

In Molène Archipelago the dominant genus in the CCA studied was Phymatolithon, while in Galicia was Mesophyllum, this latter having a southern distribution along the Atlantic European coasts (Guiry \& Guiry 2016). This gradual replacement of both species with latitude has been also observed in the composition of major maerl- 

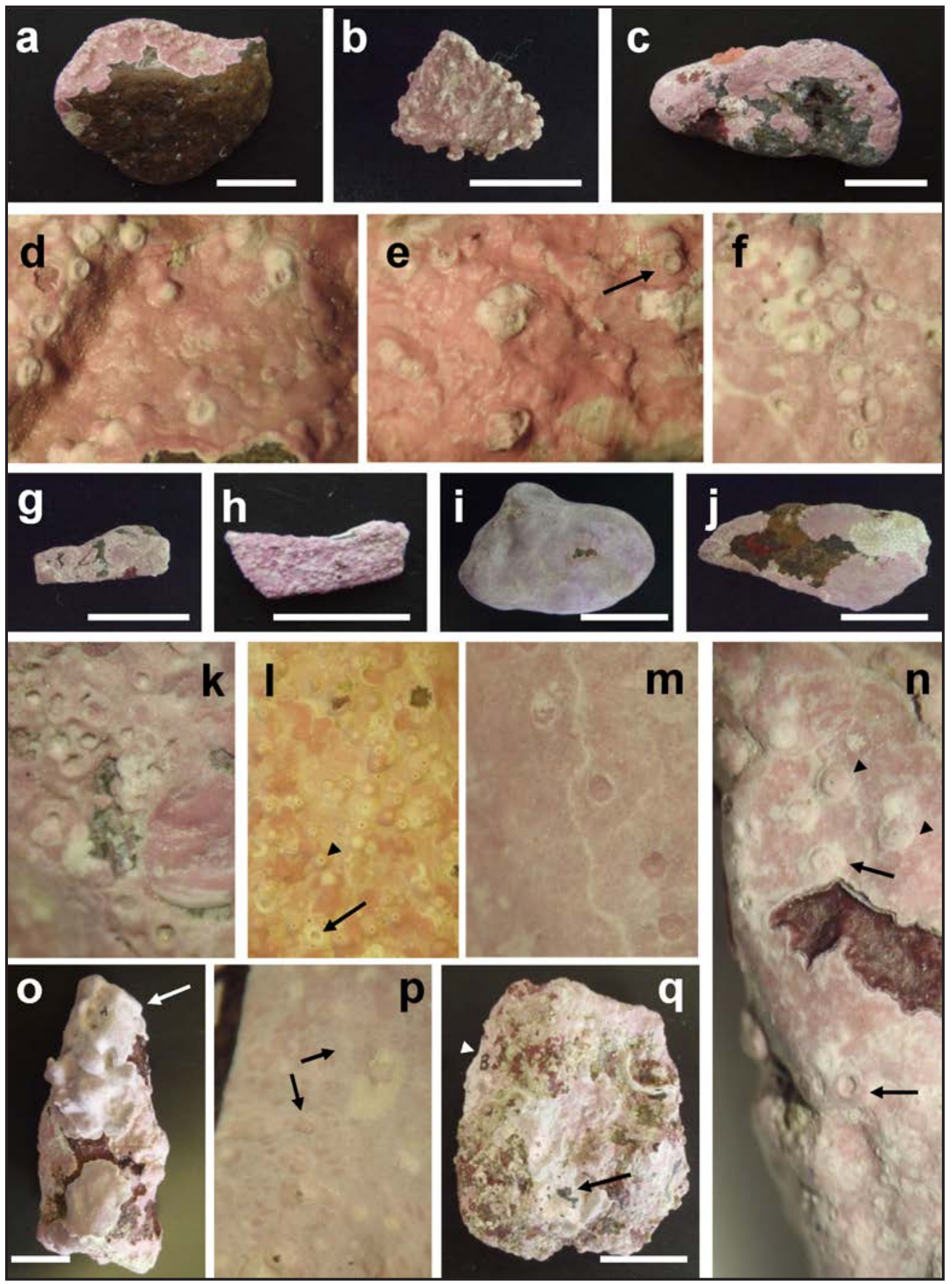

Fig. 4. Epilithic crusts associated with maerl beds in Galicia and in Brittany: a, Lithothamnion sp. 2 from Ons Archipelago (VPF00483); b, Lithothamnion sp. 2 from Ons Archipelago (CPVP-763); c, Mesophyllum sp. 3 from Ons Archipelago (VPF00479); d, Lithothamnion sp. 2 with asexual multiporate conceptacles (VPF00483); e, Lithothamnion sp. 2 with asexual multiporate conceptacles (CPVP-763); f, Mesophyllum sp. 3 with asexual multiporate conceptacles (VPF00479); g, Mesophyllum sp. 3 from Ons Archipelago (CPVP-767); h, Mesophyllum sp. 4 from Ons Archipelago (VPF00480); i, Lithophyllum sp. 3 from Ons Archipelago (CPVP-762); j, Mesophyllum sp. 4 from Ons Archipelago (CPVP-769); k, Mesophyllum sp. 3 with asexual multiporate conceptacles (CPVP-767); i, Mesophyllum sp. 4 with asexual multiporate (arrow) and sexual uniporate (arrowhead) conceptacles (VPF00480); m, Lithophyllum sp. 3 with uniporate conceptacles (CPVP-762); n, Mesophyllum sp. 4 with asexual multiporate (arrow) and sexual uniporate (arrowhead) conceptacles (CPVP-769); o, Lithophyllum hibernicum from Molène Archipelago (VPF00407A) habit; p, Lithophyllum hibernicum with uniporate conceptacles (VPF00407A); q, sample from Molène Archipelago, arrow shows Lithophyllum hibernicum (VPF00411A), arrowhead shows Phymatolithon sp. 6 (VPF00411B). [Scale bars: a, b, c, g, h, j, 1cm; i, o, q, 2 cm.] 
forming species in the OSPAR maritime area, including Brittany and Galicia (Carro \& al. 2014; Pardo \& al. 2014).

According to the literature, Phymatolithon calcareum is considered as a major maerl-forming species in Atlantic Europe (Irvine \& Chamberlain 1994; Carro \& al. 2014; Pardo \& al. 2014). Our record of CCA with uniporate sexual conceptacles in the Breton maerl bed agrees with previous reports of gametophytes - including the carposporophyte - under crustose forms, all of them from this region (Mendoza \& Cabioch 1998; Peña \& al. 2014). This observation would corroborate that, at least in Brittany, the gametangial stage of Phymatolithon calcareum occurs as associated crusts - i.e. attached-, while unattached maerl plants of this species are sporophytes (Cabioch 1969, 1970; Mendoza \& Cabioch 1998). However, in adjacent areas such as the British Isles or Atlantic Iberian Peninsula where maerl beds have been intensively studied, fertile gametophytes - crusts or unattached - have been never recorded yet (Irvine \& Chamberlain 1994; Peña \& al. 2014, 2015b). Given that all Atlantic European records of gametangial stages of Phymatolithon calcareum are still restricted to Brittany (Mendoza \& Cabioch 1998; Peña \& al. 2014), we presume that this Atlantic region plays an important role in the life-history of this major maerlforming species.

Phymatolithon lamii is a crustose species occurring from the intertidal to subtidal rocky shores of the Atlantic coasts from Arctic Norway to South Atlantic Iberian Peninsula (Chamberlain 1991; Irvine \& Chamberlain 1994; Peña \& al. 2015b). Our collection in the Breton maerl bed as associated CCA corroborated recent records of this species from the same maerl bed - Molène archipelago (Peña \& al. 2015b).

A recent molecular study (Hernández-Kantún \& al. 2015a) confirmed that Lithophyllum hibernicum is a common intertidal crust that has been repeatedly misidentified in the Atlantic European literature as Lithophyllum incrustans, which also occurs as both encrusting and maerl forms -i.e., Pardo \& al. (2014), as Lithophyllum fasciculatum (Lam.) Foslie and Lithophyllum dentatum (Kütz.) Foslie-. In our study, Lithophyllum hibernicum was recorded subtidally in the Breton maerl bed as associated CCA.

A non-common morpho-anatomical observation was detected in the Galician specimen identified as Mesophyllum sp. 4 (CPVP-769): both types of conceptacles - uniporate sexual and multiporate asexual - occurred in the same crust surface. A similar observation was found in the Galician maerl species Mesophyllum sphaericum V.Peña \& al. in Galicia (see fig. 2a in Peña \& al. 2011).

Regarding the development of new maerl plants in the Atlantic European maerl beds, two main mechanisms have been suggested in the literature: i) vegetative fragmentation
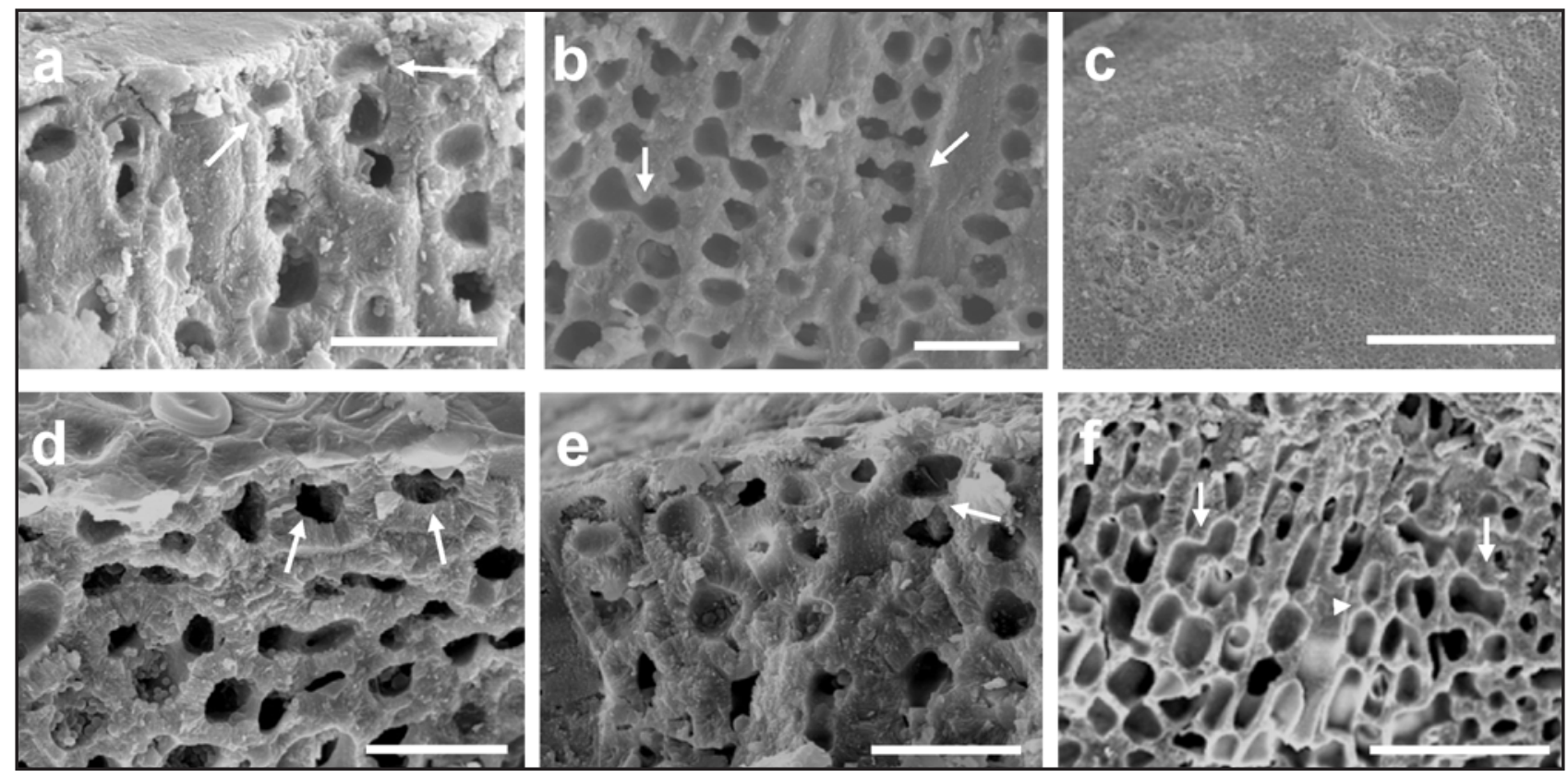

Fig. 5. Anatomical features of Phymatolithon sp. 5 (CPVP-758) and Lithothamnion sp. 2 (CPVP-763) under SEM: a, Phymatolithon sp. 5, vertical section of more or less domed epithallial cells (arrows); b, Phymatolithon sp. 5, vertical section of fusions cells (arrows); c, Phymatolithon sp. 5, surface view of multiporate conceptacles; d, Lithothamnion sp. 2, vertical section showing flared epithallial cells (arrows); e, Lithothamnion sp. 2, vertical section showing primary pit connections (arrow); f, Vertical section showing fusion cells (arrows) and primary pit connections (arrowhead). [Scale bars: a, b, d, e, $15 \mu \mathrm{m}$; c, $200 \mu \mathrm{m}$; f, $50 \mu \mathrm{m}$.] 


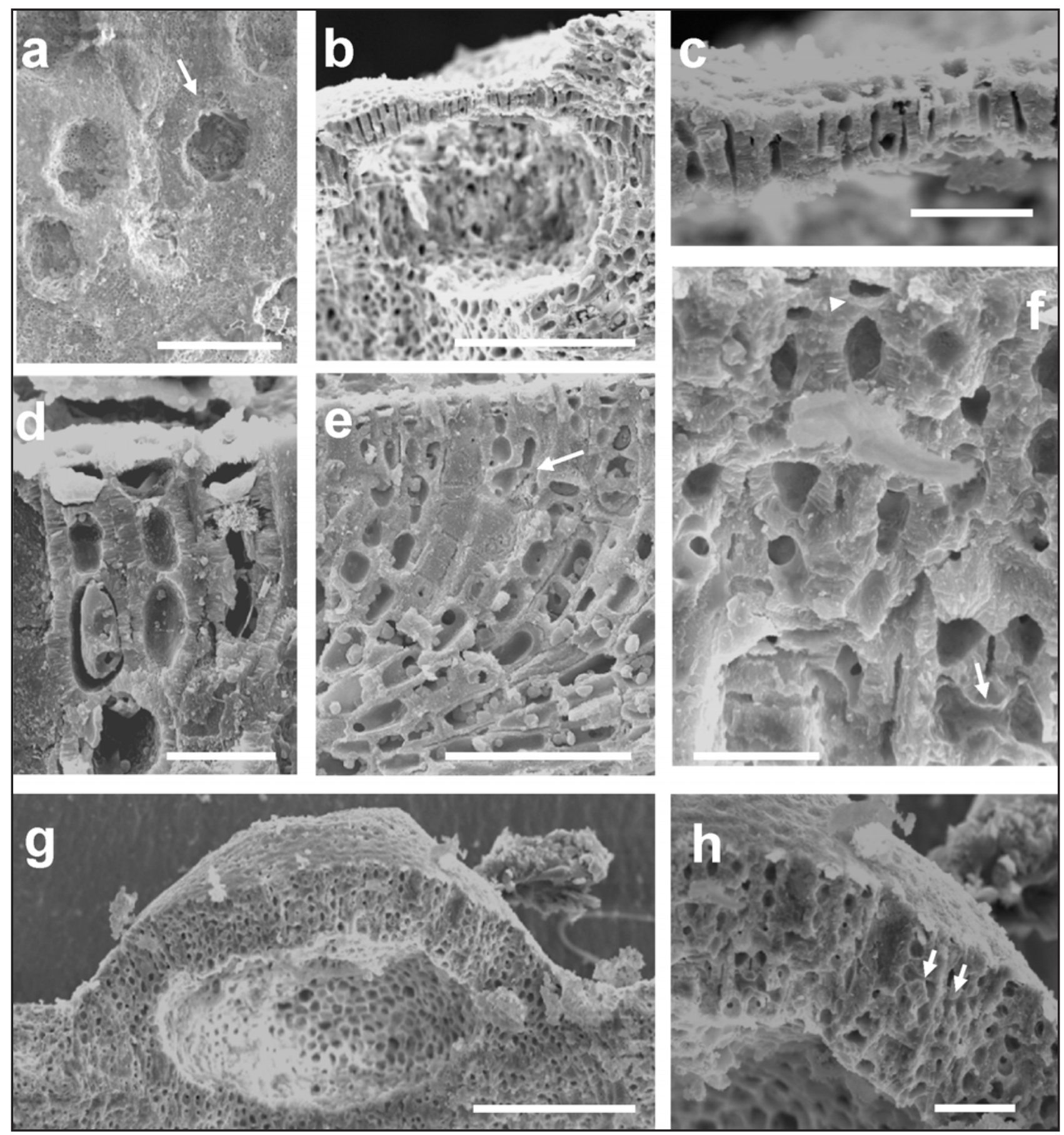

Fig. 6. Anatomical features of Mesophyllum sp. 3 (CPVP-767) and Mesophyllum sp. 4 (CPVP-769) under SEM: a, Mesophyllum sp. 3, surface view of asexual multiporate conceptacles; b, Mesophyllum sp. 3, vertical section of a asexual multiporate conceptacle; c, Mesophyllum sp. 3, vertical section showing the roof of an asexual multiporate conceptacle; d, Mesophyllum sp. 3, vertical section of somewhat flared epithallial cells; e, Mesophyllum sp. 3, vertical section showing cells fusions (arrow); f, Mesophyllum sp. 4, vertical section showing flattened epithallial cells (arrowhead) and fusion cells (arrow); g, Mesophyllum sp. 4, vertical section of an asexual multiporate conceptacle; h, Mesophyllum sp. 4, vertical section showing roof of an asexual multiporate conceptacle with fusion cells (arrows). [Scale bars: a, $250 \mu \mathrm{m}$; b, e, g, $100 \mu \mathrm{m} ; \mathrm{c}, \mathrm{h}, 20 \mu \mathrm{m} ; \mathrm{d}, \mathrm{f}, 10 \mu \mathrm{m}$. 
of own free-living maerl plants (Bosence 1976; Woelkerling 1988; Peña \& al. 2014); ii) involvement of associated CCA in the recruitment of new maerl plants - i.e., detached branches of crusts (Lemoine 1910; Cabioch 1969, 1970; Freiwald 1995; Mendoza \& Cabioch 1998)_- Our study pointed out that the latter hypothesis could be applied, but only for Phymatolithon calcareum in the Breton maerl beds, where gametangial — plus carposporangial- and sporangial stages occur under different growth-forms -CCA and maerl, respectively (Cabioch 1969, 1970; Mendoza \& Cabioch 1998; Peña \& al. 2014)—. However, in the Galician maerl beds the species composition of associated CCA in comparison with the main maerlforming taxa - Phymatolithon calcareum, Phymatolithon lusitanicum, and Lithothamnion corallioides - appeared to be different. This together with the absence of maerl species records bearing gametangial —and carposporangialstructures in this region - attached or unattached-, and the occasional records of sporangial conceptacles observations (Peña \& Bárbara 2004), the fragmentation of own free-living maerl plants should be considered as the main propagation method in the Galician maerl beds.

\section{CONCLUSION}

The present study carried out in two protected Atlantic European maerl beds pointed out that maerl beds harbours a high species diversity of associated CCA, which may not share the same species composition that maerl-forming algae. Also a high cryptic diversity was found in the associated CCA, as occurs in the maerl-forming species (Carro \& al. 2014; Pardo \& al. 2014) and in geniculate coralline algae (Walker \& al. 2009; Pardo \& al. 2015). Further extensive studies in other European maerl beds are necessary to understand this unexpected diversity of associated CCA. Nonetheless, according to our results, the crustose growth-forms should be taken into account in management actions of Atlantic European maerl beds.

\section{ACKNOWLEDGEMENTS}

This research was supported by Ministerio de Ciencia e Innovacion of Spain (CTM2010-18787, partially co-founded by FEDER, Fondo Europeo de Desarrollo Regional) and Xunta de Galicia (10MMA103003PR). Cristina Pardo acknowledges financial support by Xunta de Galicia (Axudas á etapa predoutoral do Plan I2C, 2011) and Spain's Ministerio de Educación (Programa FPU, 2010). Viviana Peña acknowledges support by Universidade da Coruña (Contrato programaCampus Industrial de Ferrol). We acknowledge to Parc Naturel Marin d'Iroise and Parque Nacional Marítimo Terrestre das Illas Atlánticas de Galicia by allowing our surveys. We are also very grateful to Jacques Grall for fieldwork collaboration in Molène Archipelago (Brittany).

\section{REFERENCES}

Adey W.H. \& Mckibbin D.L. 1970. Studies on the maerl species Phymatolithon calcareum (Pallas) nov. comb. and Lithothamnium coralloides Crouan in the Ría de Vigo. Botanica Marina 13: 100-106. https://doi.org/10.1515/botm.1970.13.2.100
Adey W., Halfar J., Humphreys A., Suskiewicz T., Belanger D., Gagnon P. \& Fox M. 2015. Subartic rhodolith beds promote longevity of crustose coralline algal buildups and their climate archiving potential. Palaios 30: 281-293. https://doi.org/10.2110/palo.2014.075

Basso D., Nalin R. \& Nelson C. 2009. Shallow-water Sporolithon rhodoliths from North Island (New Zealand). Palaios 24: 92-103. https://doi.org/10.2110/palo.2008.p08-048r

Bosence D.W. 1976. Ecological studies on two unattached coralline algae from western Ireland. Palaeontology 19: 365-395.

Cabioch J. 1969. Les fonds de maerl de la Baie de Morlaix et leur peuplement végétal. Cahiers de Biologie Marine 10: 139-161.

Cabioch J. 1970. Le maërl des côtes de Bretagne et le problème de sa survie. Penn ar Bed 7: 421-429.

Carro B., López L., Peña V., Bárbara I. \& Barreiro R. 2014. DNA barcoding allows the accurate assessment of European maerl diversity: a proof-of-concept study. Phytotaxa 190: 176-189. https://doi.org/10.11646/phytotaxa.190.1.12

Chamberlain Y.M. 1991. Observations on Phymatolithon lamii (Lemoine) Y. Chamberlain comb. nov. (Rhodophyta, Corallinales) in the British Isles with an assessment of its relationship to P. rugulosum, Lithophyllum lamii and L. melobesioides. British Phycological Journal 26: 219-233. https://doi.org/10.1080/00071619100650201

Darriba D., Taboada G.L., Doallo R. \& Posada D. 2012. jModelTest 2: more models, new heuristics and parallel computing. Nature Methods 9: 772-772. https://doi.org/10.1038/nmeth.2109

Freiwald A. 1995. Sedimentological and biological aspects in the formation of branched rhodoliths in northern Norway. Beiträge zur Paläontologie 20: 7-19.

Guiry M.D. \& Guiry G.M. [2016]. AlgaeBase. Galway, Ireland. Website: http://www.algaebase.org [Accessed: 4 November 2016].

Hall-Spencer J.M., Kelly J. \& Maggs C.A. 2010. Background document for maërl beds. OSPAR Commission Biodiversity Series, OSPAR Commission: 1-36.

Hernández-Kantún J.J., Rindi F., Adey W.H., Heesch S., Peña V., Le Gall L. \& Gabrielson P.W. 2015a. Sequencing type material resolves the identity and distribution of the generitype Lithophyllum incrustans, and related European species L. hibernicum and L. bathyporum (Corallinales, Rhodophyta). Journal of Phycology 51: 791-807. https://doi.org/10.1111/jpy.12319

Hernández-Kantún J.J., Riosmena-Rodríguez R., Hall-Spencer J.M., Peña V., Maggs C.A. \& Rindi F. 2015b. Phylogenetic analysis of rhodolith formation in the Corallinales (Rhodophyta). European Journal of Phycology 50: 46-61. https://doi.org/10.1080/09670262.2014.984347

Hernández-Kantún J.J., Riosmena-Rodríguez R., Adey W.H. \& Rindi F. 2014. Analysis of the cox $2-3$ spacer region for population diversity and taxonomic implications in rhodolith-forming species (Rhodophyta: Corallinales). Phytotaxa 190: 331-354. https://doi.org/10.11646/phytotaxa.190.1.20

Irvine L.M. \& Chamberlain Y.M. 1994. Seaweeds of the British Isles vol. 1: Rhodophyta, Part 2B Corallinales, Hildenbrandiales. The Natural History Museum, London.

Lemoine M.P. 1910. Répartition et mode de vie du Maërl (Lithothamnium calcareum) aux environs de Concarneau (Finistère). Annales de Institut Océanographique. Fondation Albert Ier, Prince de Monaco 1: 1-28.

Maggs C.A. 1983. A phenological study of the epiflora of two maerl beds in Galway Bay. PhD thesis, National University of Ireland, Galway.

Mendoza M.L. \& Cabioch J. 1998. Étude comparée de la reproduction de Phymatolithon calcareum (Pallas) Adey \& McKibbin et Lithothamnion 
corallioides (P. \& H. Crouan) P. \& H. Crouan (Corallinales, Rhodophyta), et reconsidérations sur le définition des genres. Canadian Journal of Botany 76: 1433-1445. https://doi.org/10.1139/b98-116

Pardo C., López L., Peña V., Hernández-Kantún J., Le Gall L., Bárbara I. \& Barreiro R. 2014. A multilocus species delimitation reveals a striking number of species of coralline algae forming maerl in the OSPAR maritime area. PLOS ONE 9: e104073. https://doi.org/10.1371/journal.pone.0104073

Pardo C., Peña V., Barreiro R. \& Bárbara I. 2015. A molecular and morphological study of Corallina sensu lato (Corallinales, Rhodophyta) in the Atlantic Iberian Peninsula. Cryptogamie, Algologie 36: 31-54. https://doi.org/10.7872/crya.v36.iss1.2015.31

Peña V. \& Bárbara I. 2004. Diferenciación morfológica y anatómica entre Lithothamnion corallioides y Phymatolithon calcareum (Corallinales, Rhodophyta) en dos bancos de maërl de la Ría de Arousa (N. O. Península Ibérica). Anales de Biología 26: 21-27.

Peña V. \& Bárbara I. 2009. Distribution of the Galician maerl beds and their shape classes (Atlantic Iberian Peninsula): proposal of areas in future conservation actions. Cahiers de Biologie Marine 50: 353-368.

Peña V., Adey W.H., Riosmena-Rodríguez R., Jung M.-Y., AfonsoCarrillo J., Choi H.G. \& Bárbara I. 2011. Mesophyllum sphaericum sp. nov. (Corallinales, Rhodophyta): a new maërl-forming species from the northeast Atlantic. Journal of Phycology 47: 911-927. https://doi.org/10.1111/i.1529-8817.2011.01015.x

Peña V., Hernández-Kantún J.J., Grall J., Pardo C., López L., Bárbara I., Le Gall L. \& Barreiro R. 2014. Detection of gametophytes in the maerl-forming species Phymatolithon calcareum (Melobesioideae, Corallinales) assessed by DNA barcoding. Cryptogamie, Algologie 35: 15-25. https://doi.org/10.7872/crya.v35.iss1.2014.15

Peña V., De Clerck O., Afonso-Carrillo J., Ballesteros E., Bárbara I., Barreiro R. \& Le Gall L. 2015a. An integrative systematic approach to species diversity and distribution in the genus Mesophyllum (Corallinales, Rhodophyta) in Atlantic and Mediterranean Europe. European Journal of Phycology 50: 20-36. https://doi.org/10.1080/09670262.2014.981294
Peña V., Pardo C., López L., Carro B., Hernández-Kantún J., Adey W.H., Bárbara I., Barreiro R. \& Le Gall L. 2015b. Phymatolithon lusitanicum sp. nov. (Hapalidiales, Rhodophyta): the third most abundant maerlforming species in the Atlantic Iberian Peninsula. Cryptogamie, Algologie 36: 1-31. https://doi.org/10.7872/crya/v36.iss4.2015.429

Saunders G.W. 2005. Applying DNA barcoding to red macroalgae: a preliminary appraisal holds promise for future applications. Philosophical Transactions of the Royal Society B: Biological Sciences 360: 1879-1888. https://doi.org/10.1098/rstb.2005.1719

Saunders G.W. \& McDevit D.C. 2012. Methods for DNA barcoding photosynthetic protists emphasizing the macroalgae and diatoms. Methods in Molecular Biology 858: 207-222. https://doi.org/10.1007/978-1-61779-591-6 10

Tamura K., Stecher G., Peterson D., Filipski A. \& Kumar S. 2013. MEGA6: Molecular Evolutionary Genetics Analysis version 6.0. Molecular Biology and Evolution 30: 2725-2729. https://doi.org/10.1093/molbev/mst197

Thiers B. [2016]. Index Herbariorum: A Global Directory of Public Herbaria and Associated Staff. New York Botanical Garden's Virtual Herbarium. Website: http://sweetgum.nybg.org/science/ih/ [Accessed: 4 November 2016].

Walker R.H., Brodie J., Russell S., Irvine L.M. \& Orfanidis S. 2009. Biodiversity of coralline algae in the Northeastern Atlantic including Corallina caespitosa sp. nov. (Corallinoideae, Rhodophyta). Journal of Phycology 45: 287-297. https://doi.org/10.1111/j.1529-8817.2008.00637.x

Woelkerling W.J. 1988. The Coralline Red Algae: An Analysis of the Genera and Subfamilies of Nongeniculate Corallinaceae. Oxford University Press, London.

Woelkerling W.J. \& Irvine L.M. 1986. The typification and status of Phymatolithon (Corallinaceae, Rhodophyta). British Phycological Journal 21: 55-80. https://doi.org/10.1080/00071618600650071 